\title{
Massive pre-main-sequence stars in M17
}

\author{
M. C. Ramírez-Tannus ${ }^{1}$, L. Kaper, A. de Koter, F. Tramper, \\ H. Sana, O. H. Ramírez-Agudelo, A. Bik, L. E. Ellerbroek \\ and B. B. Ochsendorf
}

${ }^{1}$ API, University of Amsterdam, The Netherlands; email: m.c.ramireztannus@uva.nl

We obtained VLT/X-shooter spectra of twelve candidate young massive stars previously selected by Hanson et al. (1997) in the giant Hir region M17. An analysis of their spectra using FASTWIND models (Puls et al. 2005) shows that they span a mass range of $6-20 \mathrm{M}_{\odot}$. We identify the presence of gaseous and dusty disks around six sources based on emission lines in the spectrum and infrared continuum excess. By comparing their position in the HRD with theoretical PMS tracks we conclude that these are genuine pre-main-sequence (PMS) stars contracting towards the main sequence after having experienced high mass-accretion $\left(M_{\text {acc }} \sim 10^{-4}-10^{-3} \mathrm{M}_{\odot} \mathrm{yr}^{-1}\right)$. Three sources are close to the ZAMS, for two objects we did not detect a gaseous disk, and one object might be a PMS star without a disk. Though the sample is small, it does allow for a first analysis of statistical properties of a massive PMS star population (Ramírez-Tannus et al. 2017, A\&A, in press). We find a strikingly small radial velocity dispersion, which is at odds with similar distributions of few-Myr-old OB star populations. This may either point to M17 having a low binary fraction $\left(f_{b i n}=0.12\right)$ or to a lack of short period binaries (in our sample $P_{\text {cutoff }}>9$ months) relative to these older populations (Sana et al. 2017), characterised by $\mathrm{f}_{\text {bin }}=0.7$ and $P_{\text {cutoff }}>1.4$ days (Sana et al. 2012).
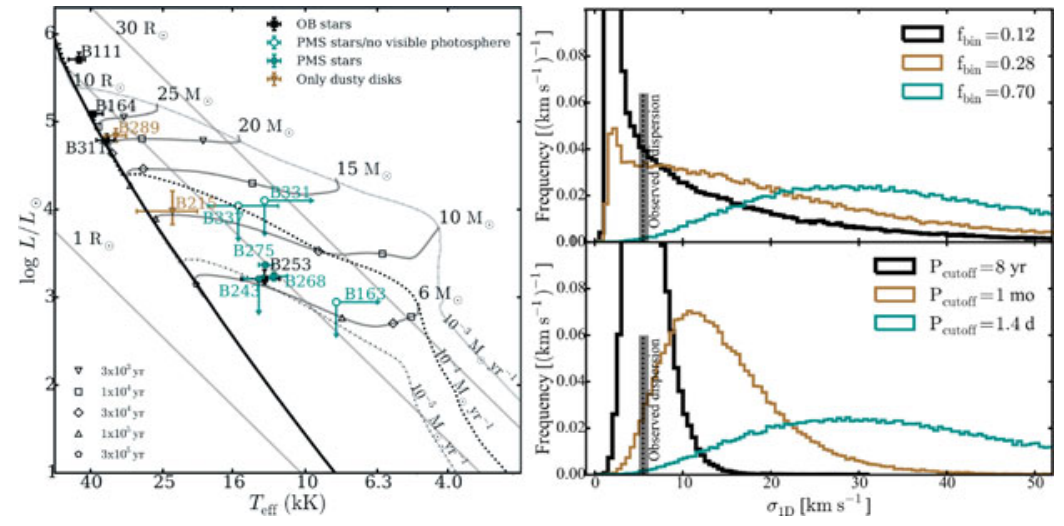

Figure 1. Left: HRD for our sources in M17. The light-blue dots show the PMS stars surrounded by gaseous and dusty disks, the black squares the OB stars, the brown triangles objects surrounded by dusty disks. The filled symbols represent the stars that we modelled with FASTWIND and the open symbols represent the objects for which we derived $T_{\text {eff }}$ and $L / L \odot$ from their spectral type (from Ramírez-Tannus et al. 2017). Right: Simulated $\sigma_{1 \mathrm{D}}$ : Top: For different binary fractions, we discard $f_{\text {bin }}>0.34$ and $>42$ at the 10 and $5 \%$ level, respectively. Bottom: For different cutoff periods, we discard $P_{\text {cutoff }}<131$ and $<47$ days at the 10 and $5 \%$ level, respectively. The vertical line represents the observed $\sigma_{1 \mathrm{D}}$ and the associated error for our M17 stars (from Sana et al. 2017).

\section{References}

Hanson, M. M., Howarth, I. D., \& Conti, P. S. 1997, ApJ, 489, 698

Hosokawa, T. \& Omukai, K. 2009, ApJ, 691, 823

Puls, J., Urbaneja, M. A., Venero, R., et al. 2005, A\&A, 435, 669

Sana, H., de Mink, S. E., de Koter, A., et al. 2012, Science, 337, 444

Sana, H., Ramirez-Tannus, M., de Koter, A., \& Kaper, L., et al. 2017, ArXiv:1702.02153 